\title{
On the Nature of the Verb in Present Day English (Existential) There-Constructions. Formal and Communicative Implications
}

\author{
Ana E. Martínez Insúa \\ University of Santiago \\ iaminsua@usc.es
}

\begin{abstract}
There is general agreement that $b e$ is the verb 'par excellence' in so-called English existential there-constructions (existential TCs). In fact, on many occasions, a distinction is made between existential TCs -be TCs- and presentational TCs (non-be TCs). It is true, however, that other verbs may also occur in TCs. These are mainly intransitive verbs that express the ideas of permanence, being, existence, movement, position, entrance into the scene of discourse, arrival or continuation of an event (seem, come, remain, follow, develop, etc.).

From a corpus-driven perspective, the proportion of non-be TCs in Present Day English (PDE) is analysed, granting special attention to their communicative value. It is hypothesised that even though, strictly speaking, non-be TCs do not primarily express existence, they do share with be TCs their main pragmatic function -i.e. signal function- a trait genuinely linked to the use of there. It is also examined whether any significant difference is observed between the use of non-be TCs in speech and writing, and what is the rationale for this difference (if any).
\end{abstract}

\section{Introduction ${ }^{1}$}

The question of the verb occurring in so-called existential there-constructions (hereafter, existential TCs) has been a common topic in the recent literature. It is, certainly, one of the most characteristic features of the constructions containing existential there, and most -if 
not all- relevant approaches to TCs refer to the nature of the verb they contain (Erdmann, 1976: 135ff; Bolinger, 1977: 96ff; Milsark, 1979: 245ff; Huddleston, 1984: 469; Hannay, 1985: 9ff; Quirk et al., 1985: $\$ 18 \bullet 49$; Lakoff, 1987: 550, 572ff; Breivik, 1990: 157ff; Downing and Locke, 1992: 224; Johansson, 1997: 304ff; McNally, 1997: 199; Biber et al., 1999: 944ff; Pérez Guerra, 1999: 72ff; among others). There is general agreement that $b e$ is, by far, the most commonly found verb in TCs, since the existential nature of these constructions and the requirements derived from it are best met by "transparent" verbs such as be (Pérez Guerra, 1999: 83).

In spite of that, together with be, other verbs expressing being, permanence, occurrence, movement, position, arrival, entrance into the scene of discourse, or even the continuation of an event do occur together with so-called existential there. The majority of these verbs are intransitive and are generally grouped, on semantic grounds, as verbs of being and occurrence -e.g. come, exist, hang, happen-, temporal verbs -e.g. arise, burst, emerge, loom-, verbs of continuation-develop, remain, linger, persist-, and verbs of motion -e.g. arrive, enter, come, pass- (cf. Erdmann, 1976: 137ff; Pérez Guerra, 1995: 228ff). Some scholars prefer not to include non-be TCs in the existential group, but instead to categorise them as presentative TCs (Hannay, 1985), or analogical constructions to the existential pattern in which there is a mere expletive subject-filler (Pérez Guerra, 1999). ${ }^{2}$ This question will be revisited in section 3 below.

Adopting a corpus-driven perspective, ${ }^{3}$ and starting with a mandatory look at naturally produced TCs in context, this paper seeks to gain some deeper insight into the nature of the verbs that occur in PDE TCs, and their communicative roles. The medium of expression (written or spoken) will be taken into consideration as a factor possibly related to the frequencies and distribution of the different verbs under analysis.

The paper will be, therefore, organised as follows. Section 2 will explain the methodology employed for the design of the corpus and the retrieval of the examples. Section 3 will briefly outline the treatment of non-be TCs in the recent literature. After that, sections 4 and 5 will examine the findings and to the general structural features of the TCs under analysis. Section 6 will deal with the communicative functions that $b e$ and non-be TCs present when studied in context, including a personal understanding of their properties, both common and distinctive. Finally, section 7 will attempt to draw some general conclusions from the analysis of the data, based on the findings of the previous sections.

The study here presented is based on the analysis of the TCs found in a one-millionwords subcorpus of the British National Corpus (BNC). Among these examples (a total of $2690 \mathrm{TCs}$ ), 10 non-be TCs were registered, containing forms of the verbs follow, come, remain, develop and decipher. For the sake of brevity, these ten examples are given here as (1)-(10); in the following pages I will refer to them using only their numbers.

And and even there will come a time after the private dialogue, where the people have the right (K6A 435 sp, leisure). ${ }^{4}$

But there will come a time when you may have to think about it (G4G 268, sp, public and institutional). 
Now surely if you're gonna intern the leaders and m might they include Gerry Addams and we we could talk about the other side, we could talk about the Loyalist er paramilitaries equally, surely there's just another wave gonna come along because it's a never ending assembly line of terrorists, so what's the point? (HV2 $138 \mathrm{sp}$, leisure).

And I think that there's going to come a point (K6A $373 \mathrm{sp}$, leisure).

Oh yes varied, and there interesting, with, with children, particularly if they erm if I' $m$ doing workshops er with art and writing workshops and we come across an abstract picture, the children music, and, we, as a way into their vocabulary which isn't as large as their, their writing skills, and there'll, there'll decipher an abstract picture, wonderful language and I like doing that you know (F71 $110 \mathrm{sp}$, educational and informative).

Because there must come a point somewhere in time where the shop will say well you've had these goods so long they're nolonger our responsibility (FUT $334 \mathrm{sp}$, public and institutional).

There remains the possibility of incised carved lettering of course (A0X $1538 \mathrm{wt}$, leisure).

There followed lean years in a cottage in Sussex, "living on nothing but spaghetti" while he perfected his newly-discovered skill (A0X $1628 \mathrm{wt}$, leisure).

In Britain there has developed a learned debate about the impact of party on government (A6F $17 \mathrm{wt}$, world affairs).

Everyone accepts that pits will close but there seems little change of the government being deflected by another rehellion among Tory MPs (K6F 32, sp, leisure).

Before proceeding with the analysis of these examples, it will be helpful to examine the methodology that was used to carry out this research.

\section{Method. The corpus and the TCs analysed}

The examples analysed in this study were drawn from a one-million-word sample of machine-readable running texts of contemporary English (both written and spoken). This sample is made up of texts selected from those contained in the $\mathrm{BNC}^{5}$, and therefore, should be considered a subcorpus. The subcorpus was designed to constitute a so-called disproportionally stratified sample (Butler, 1985), given that this kind of sample creates the optimum situation for the comparison of subgroups. As stated by Butler (1985), it avoids the possibility that those types of unit with a small overall proportion in the population (the $\mathrm{BNC}$, in this case) are not represented in the sample at all. Consequently, all the different genres included in the BNC, even those that contain quite a reduced number of words, have a proportional representation in the subcorpus employed in this study. ${ }^{6}$

It is true that the subcorpus is meant to maintain the main general features of the $\mathrm{BNC}^{7}$ however, apart from the obvious difference in corpus size, certain other variations were introduced, with the aim of ensuring that the sample of PDE was representative and reliable, and that the data employed for the study were as up-to-date as possible. The texts selected belong to the period that goes from 1989 to the present day, equal proportions of words were taken for both oral and written texts (half a million words for the spoken sample and 
half a million words for the written one-, and the different genres, which are represented in different proportions within the BNC, were made equal in the subcorpus here analysed. ${ }^{8}$

Once the subcorpus was selected, with the help of the tools that the BNC itself provides, the TCs contained in it were identified, counted and entered in a database. This database includes every occurrence of the existential marker there in the samples under analysis, together with a small number of cases (representing less than $1.5 \%$ of the total number of TCs- in which there is not explicit, but omitted, due to its presence in the immediately previous co(n)text (Bolinger, 1977: 114; Pérez Guerra, 1999: 77-78). Furthermore, the initial group of all the TCs found underwent a multi-stage sampling process, and was subsequently reduced, as it would have been impossible to analyse some of the examples found.

In some cases, the BNC provides the labels $\langle$ unclear $\rangle,\langle/\rangle$, or $\langle$ end of the tape $\rangle$ to indicate that it was not possible to transcribe some (fundamental) part of the utterance. (11)-(14) below exemplify this. Most of the examples of such labels were found in the spoken component of the subcorpus. This is not completely unexpected, since it is in speech where utterances frequently overlap, speakers may interrupt each other, noises may interfere, and sentences often remain unfinished.

You know snip ribbons and things I dunno, I'm not suggesting will you find out now, but there's something < unclear >. (F7C $1142 \mathrm{sp}$, business).

I think there's a, there's <unclear>. (F7C 1622 sp, business).

But if there was an aspect of your steering like erm <end of the tape> (FM0 $827 \mathrm{sp}$, business).

Er are we all agreed then we'll put down that there's a third $</>$ (F7J 418 sp, business).

After all the reductions, the group of TCs that were considered for the present investigation amounts to 2690: 1071 TCs taken from the written sample, and 1619 TCs from the spoken one. In order to classify them according to the verbal form they contain, attention was first devoted to main verbs, and then to the presence of modals, semi-modal auxiliaries or catenative constructions together with them.

In the tables that will be provided in section 4 , four main groups will be distinguished: main verbs, central modals, catenative constructions, and semi-modals. Following Biber et al. (1999: 483-ff), The category labelled semi-modals will include cases of marginal auxiliary verbs -need to, ought to, used to-, fixed idiomatic phrases -be going to, gonna, have got to, gotta, be to-, and other relatively fixed expressions such as be likely to. ${ }^{9}$ Admittedly, the boundaries between these groups are not clear cut and they need to be considered as forming part of a continuum, yet it is necessary to establish some kind of classification. 


\section{The verb in TCs. Some necessary considerations}

For the sake of clarity in the subsequent discussion, it seems appropriate to say a few words about the dichotomy $b e$ TCs vs. non-be TCs, and the way in which it has been approached in previous research.

As mentioned in the introduction, most -if not all- approaches to TCs refer to the somehow marginal character of non-be TCs. The general conclusion is, in Biber et al.'s words, that "overall, although combinations occur with other verbs, existential clauses are overwhelmingly associated with the verb be" (1999: 946). Similarly, there is general agreement that, in these cases of there + verb (V) other than be, "the notion 'presenting on the scene' as the discourse function of there-structures is more appropriate than that of expressing "existence'" (Downing and Locke, 1992: 260). In fact, there is a tendency to apply the term existential only to TCs containing be as their main verb. In Hannay (1985: 9-ff), for instance, a number of differences between be and non-be TCs are reported in order to support the distinction between existential presentatives (be TCs) and there presentatives (non-be TCs). A similar terminological distinction is established in Birner and Ward (1998) between existential and presentational TCs. Furthermore, non-be TCs containing full lexical verbs are sometimes characterised "as [a] construction[s] analogical to the existential pattern" (Pérez Guerra, 1999: 86), rather than archetypal existential constructions, since they do not really mark the entity in the post-verbal noun phrase (PVNP) as existing.

In brief then, what is concluded and initially accepted in the present study is that, more than primarily expressing existence, non-be TCs organise their constituents according to the there + verb $(\mathrm{V})+$ notional subject order for communicative and pragmatic reasons. Initially, this view is shared in the present study. It will be my aim to try to gain some insight into the effects that such an organisation of the information has on the overall communicative value of the statements. It will be contended that the pragmatic role of there (its pointing and signal function, cf. Curme, 1966: 100; or Breivik, 1990: 163, 175) is a characteristic of non-be TCs too. These seem to trigger certain pragmatic effects that are not so evident in $b e$ TCs (i.e. to signal not only the notional subject but also the process). To the best of my knowledge, despite some allusions to this can be found in the literature, not much attention has been granted to the communicative effects that non-be TCs have as they are used in PDE. This may be due to their remarkably low frequency of occurrence both in writing and speech, which will be shown in section 4 below.

\section{Results}

This section will provide the tables with the data derived from the analysis of the corpus. It will also contain some brief comments on the findings, although a lengthier analysis of the structural and communicative features of the TCs under analysis will be given in sections 5 and 6 below. As outlined in section 2 , in order to analyse the nature of the verbal 
constituent of TCs, first of all I will examine the frequency and distribution of the main verbs. These, and specially other-than-be verbs, will be the main focus of analysis in the following sections. Even if the presence of modals and catenative constructions in TCs is not really outstanding, the tables with their frequency and distribution will be also provided, with the aim to establish some kind of parallelism or contrast between their general use in language and their use in the specific case of TCs.

\subsection{Main verbs}

Like in previous studies (Erdmann (1976); Breivik (1990); Pérez Guerra (1999) to mention just a few), and in agreement with Bolinger's (1977: 102) claims, sentences where there cooccurs with a transitive verb in the active voice turned out to be extremely rare in the analysis of the subcorpus employed here.

As expected, be turned out to be the predominant main verb occurring in the TCs of both samples of PDE. It occurs as the main verb in $99.71 \%$ of the TCs of the written sample, and $99.63 \%$ of those in the spoken one. This results in a total average of $99.66 \%$. Notice that in a small number of examples, the sequence there $+\mathrm{V}$ was omitted due to its presence in the immediate preceding context, that is, in a previous TC (cf. Bolinger, 1977: 114, or Pérez Guerra, 1999: 77-78). In such cases, given that the verb present in the first TC was $b e$, it was assumed that the omitted one was also be. Therefore, in Table 1, they were counted as members of the be category.

\begin{tabular}{|l|c|c|c|c|c|c|}
\hline Main verb & \multicolumn{2}{|c|}{ Written sample } & \multicolumn{2}{c|}{ Spoken sample } & \multicolumn{2}{c|}{ Total } \\
\hline Be & No. & $\%$ & No. & $\%$ & No. & $\%$ \\
Come & 1068 & 99.71 & 1613 & 99.63 & 2681 & 99.66 \\
Decipher & & & 5 & 0.30 & 5 & 0.18 \\
Seem & & & 1 & 0.06 & 1 & 0.04 \\
Follow & & & 1 & 0.06 & 1 & 0.04 \\
Remain & 1 & 0.09 & & & 1 & 0.04 \\
Develop & 1 & 0.09 & & & 1 & 0.04 \\
\hline
\end{tabular}

Table 1: Main verbs in the TCs under analysis.

Table 1 evinces the low frequency of non-be TCs. Not even $1 \%$ of the TCs here analysed contained a lexical verb other-than-be as their main verb (in clear contrast with the overwhelming $99.66 \%$ of the cases, in which $b e$ is the main verb). Among the verbs found in the spoken sample, we find $b e$ (or $b e$ in combination with some auxiliary verb), come, and seem. Following Erdmann's (1976: 140ff) classification, they are verbs of being (be, seem) and occurrence (come), which express ideas equivalent to be, be visible/apparent, and occur when they are used with event and time nouns. It is important to mark here that seem occurs in TCs both, as a main verb (example (10) above) or inserted in catenative verbal constructions (example (15) below), being the latter case considerably more 
common. Both possibilities, and similar differences in their frequency of occurrence, are in fact attested by previous research (cf., for instance, Breivik, 1990: 160; Biber et al: 1999: 946).

Also in the spoken sample, a case of there followed by the transitive verb decipher was found. Despite its marginality and oddity, this example was not excluded from the frequency counts and it is here reproduced as (5). This will be examined in further detail in sections 5 and 6 below.

With regard now to the written sample, the group of non-be TCs attested is less numerous. Only three examples were registered, in contrast with the seven non-be TCs found in the spoken sample. All the three written non-be TCs contain what Erdmann calls verbs of continuation, and express the ideas of permanence and continuation. Two of these verbs, follow in (8) and develop in (9), may be used both, as transitive and intransitive verbs, being their intransitive use the one exemplified in the TCs.

Strictly speaking, from a communicative point of view, these verbs do not seem to mark the (non-)existence of the entity in the PVNP in the first place. That is not their primary function, but it is also true that all of them -with the possible exception of decipher-do transmit ideas that are closely related to the idea of (coming into) existence. I refer here to the above mentioned ideas of occurrence and entrance into the scene -in there + come-, being and permanence -in there + seem/remain- or continuation -in there + develop/follow-. What is in fact common to all of them, be and non-be TCs, is the signal function that the use of existential there conveys. Taking for granted that TCs are used for introducing new information into the communicative process without transgressing the principle of end-weight and the given-before-new principle, and the fact that there pragmatically functions as a presentative signal, TCs allow the notional subject to receive a foregrounded interpretation, so it "becomes the communicative core" (Breivik, 1990: 175-176).

Admittedly, the frequencies of non-be TCs registered in the corpus analysed here are remarkably low, and cannot be presented as arguments for any claim that the use of there is productive in order to convey a communicative signal function with other-than-be verbs. However, the fact that the number of non-be TCs found in this corpus is slightly higher in the spoken sample than in the written one seems to imply that the use of the TC as a structural resource for achieving a specific communicative effect -i.e. signal effect- remains more productive in speech than in writing. In this line, it should be borne in mind that the study of spoken language may be considered to provide a more precise and faithful image of the actual use of language, since the written medium tends to be more conservative. Furthermore, the fact that two out of the three non-be $\mathrm{TCs}$ found in the written sample belong to a periodical of the genre labelled "leisure", which is, in principle, characterised by an informal style, seems to somehow back up this theory.

Notwithstanding that, the modest size of the corpus employed for this study, and the fact that the findings achieved somewhat differ from those reported in previous research (cf. Breivik, 1990: 249 and Biber et al (1999: 946, for instance, who found considerably lower frequencies of non-be TCs in speech than in writing) suggest the necessity to carry out 
further and lengthier research on this topic. The limitations of space, however, prevent us from undertaking such a task here.

The following sections will endeavour to show that it is precisely this signal function that non- $b e$ TCs seem to gain from the use of there. Thus, using there in initial position, the nonbe TCs under analysis do also draw the addressee's attention towards their notional subjects, even if they do not primarily mark the subject as existing, but rather as following, coming, remaining, or developing. It follows that in non-be TCs the signal function carried by there does not focus exclusively on the notional subject, but rather on both, the main verb and the notional subject. This point will be further discussed in section 6 , when dealing with the communicative functions of non- $b e$ TCs.

\subsection{Catenative verbal constructions, central modals, and semi-modals}

Given that the present study approaches the nature of the verbal component of TCs, the presence of auxiliaries cannot be disregarded, even though their frequency of occurrence is not very high (11.06\%). In this section I will focus on the different types of auxiliary and semi-auxiliary verbs that were found in the TCs, independently from the main verb they contain. This section will seek then to ascertain whether the use of these verbs in TCs is different from their general use in language or not.

The different forms under consideration will be distributed into three tables here. Table 2 will show the frequency and distribution of the catenative constructions found in the corpus analysed. The -relatively more common- presence of central modals will be illustrated in Table 3, while Table 4 will display the figures for the use of semi-modal expressions, including marginal auxiliaries, fixed idiomatic phrases and relatively fixed expressions (cf. Section 2).

\begin{tabular}{|l|c|c|c|c|c|c|}
\hline $\begin{array}{l}\text { Catenative } \\
\text { construction }\end{array}$ & \multicolumn{3}{|l|}{ Written sample } & Spoken sample & \multicolumn{2}{c|}{ Total } \\
\hline Seem to & No. & $\%$ & No. & $\%$ & No. & $\%$ \\
Happen to & 12 & 1.12 & 3 & 0.18 & 15 & 0.56 \\
Appear to & 4 & 0.37 & & 0.12 & 2 & 0.07 \\
Continue to & 1 & 0.10 & & & 4 & 0.14 \\
Total & 17 & 1.59 & 5 & 0.31 & 1 & 0.03 \\
\hline
\end{tabular}

Table 2: Catenative verbal constructions in the TCs under analysis

The study of the TCs of both samples revealed that main verbs may also occur in their toinfinitive form as complements of a lexical verb with the force of a hedge (cf. Biber $e t$ al., 1999: 944). This is the case of there-structures containing catenative constructions such as happen to be, seem to be, appear to be ${ }^{10}$ (Table 2). In these cases, the presentative and/or existential meaning of the statement often becomes softened. 
There seems to be a dearth of good small tools across the board. (A0X $1066 \mathrm{wt}$, leisure).

There appears to be a growing dissatisfaction with Rag in several universities across the country. (BPE $151 \mathrm{wt}$, leisure).

In Nonway and Denmark there continue to be high levels of popular support for state-provided welfare but (there has been a marked...) (A6F $1454 \mathrm{wt}$, world affairs).

In the case of continue to be (17) the semantic nuance emerging from the use of a catenative expression is not exactly that of softening the existential meaning, but rather reinforcing it, or transferring into it the reiterative connotations of continue. The commonalties to these clauses with catenatives arise not exclusively from their semantic features, but also from the structural ones.

As shown in Table 2, catcnative constructions were more frequently found in the TCs of the written sample. This tendency was also detected in the case of central modals, where once more the variant of medium of expression secms to have a slight influence on their frequency, as can be observed in Table 3 .

\begin{tabular}{|l|c|c|c|c|c|c|}
\hline Central modal & \multicolumn{2}{|c|}{ Written sample } & \multicolumn{2}{c|}{ Spoken sample } & \multicolumn{2}{c|}{ Total } \\
\hline & No. & $\%$ & No. & $\%$ & No. & $\%$ \\
Will & 28 & 2.61 & 33 & 2.03 & 61 & 2.26 \\
'll & 2 & 0.18 & 17 & 1.05 & 19 & 0.70 \\
Won't & & & 6 & 0.37 & 6 & 0.22 \\
Would & 13 & 1.21 & 15 & 0.92 & 28 & 1.04 \\
Wouldn't & & & 2 & 0.12 & 2 & 0.07 \\
'd & 2 & 0.18 & 2 & 0.12 & 4 & 0.14 \\
Should & 11 & 1.02 & 13 & 0.80 & 24 & 0.89 \\
Shouldn't & & & 3 & 0.18 & 3 & 0.11 \\
May & 15 & 1.40 & 10 & 0.61 & 25 & 0.92 \\
Might & 9 & 0.84 & 12 & 0.74 & 21 & 0.78 \\
Can & 4 & 0.37 & 2 & 0.12 & 6 & 0.22 \\
Could & 10 & 0.93 & 9 & 0.55 & 19 & 0.70 \\
Can't & & & 1 & 0.09 & 1 & 0.03 \\
Cannot & 1 & 0.09 & & & 1 & 0.03 \\
Must & 5 & 0.46 & 10 & 0.61 & 15 & 0.55 \\
Total & 100 & 9.33 & 135 & 8.33 & 235 & 8.73 \\
\hline
\end{tabular}

Table 3: Central modals in the TCs under analysis.

Undoubtedly, some of you will be, but there must be one or two people who are actually dreading Christmas er bearing in mind that they've had to ask somebody whom they don't really want in their house, or perhaps that person or people have asked themselves, and it's very difficult to know how to refuse isn't it really? (HM4 $415 \mathrm{sp}$, leisure).

but she thinks there could be a lot of improvement around college like the mobiles they could be in better condition. (F8M248 sp, educational and informative).

(I) if, erm while this is going round you have any questions, now would be, now would be a good time to ask if you have any, there will be time at the end to talk, you can do $<$ unclear>. (F71 $49 \mathrm{sp}$, educational and informative). 
(2) Everybody accepts there should be a staff comment on. (F7F $708 \mathrm{sp}$, educational and informative).

\begin{tabular}{|l|c|c|c|c|c|c|}
\hline Marginal auxiliary & \multicolumn{2}{|c|}{ Written sample } & \multicolumn{2}{c|}{ Spoken sample } & \multicolumn{2}{c|}{ Total } \\
\hline Used to & $\mathrm{No}$ & $\%$ & $\mathrm{No}$ & $\%$ & $\mathrm{No}$ & $\%$ \\
\hline Need to & 1 & 0.10 & 1 & 0.06 & 2 & 0.08 \\
\hline Ought to & 3 & 0.28 & & & 3 & 0.12 \\
\hline 's going to & & & 3 & 0.18 & 3 & 0.12 \\
\hline 's gonna & & & 7 & 0.43 & 7 & 0.26 \\
\hline Is going to & & & 5 & 0.30 & 5 & 0.19 \\
\hline 's got to & & & 4 & 0.24 & 4 & 0.15 \\
\hline Has to & & & 3 & 0.18 & 3 & 0.12 \\
\hline Was going to & & & 2 & 0.18 & 3 & 0.12 \\
\hline Was to & 1 & 0.10 & 2 & 0.12 & 3 & 0.08 \\
\hline ss gotta & & & 1 & 0.06 & 1 & 0.04 \\
\hline Has got to & 1 & 0.10 & 1 & 0.06 & 2 & 0.08 \\
\hline Are going to & & & 1 & 0.06 & 1 & 0.04 \\
\hline Is to & 1 & 0.10 & & & 1 & 0.04 \\
\hline Be likely to & 5 & 0.47 & & & 5 & 0.19 \\
\hline Be bound to & & & 1 & 0.06 & 1 & 0.04 \\
\hline Total & 12 & 1.12 & 34 & 2.10 & 46 & 1.71 \\
\hline
\end{tabular}

Table 4: Semi-modals in the TCs under analysis.

There used to be 'chicken and egg' situation that complicated the provision of appropriate services for ethnic minority elderly people (CGD $643 \mathrm{wt}$, social science).

There needs to be a sequential strategy in which the mother makes sure that the child is correctly oriented (CGT 633 wt, applied science).

Well, that night everyone went to sleep because if there was going to be a wedding everybody in the village would surely have to go. (F72 $129 \mathrm{sp}$, educational and informative).

Whenever an elderly family member is admitted there is likely to be some anxiety for all involved (CGD $1805 \mathrm{wt}$, social science).

In general, we can say that the variety of modals and semi-modals in the TCs analysed is wide, although their frequency is not remarkable (none of these different verbs have a frequency higher than $3 \%$ in the corpus under analysis). The usage of such forms in the TCs analysed here is, in most cases, in agreement with the tendencies observed for general language in previous studies (cf. Biber et al., 1999, for instance).

Thus, although modals present a slightly lower frequency of occurrence in spoken TCs (8.33\%) than in written ones $(9.33 \%$ ) (while Biber et al., 1999: 486-487 found higher frequencies of modals in conversation than in written texts), there seems to be similar tendencies in the use of modals in TCs and language in general. As goes for language in 
general, in the case of TCs, will and would are the most common modals, both in speech and writing. The different forms of the semi-modal be going to, by contrast, are the commonest in speech. Hence, while in spoken TCs forms of be going to are used for the expression of immediate futurity, in writing the preference is for TCs to express futurity by means of the central modal will ('ll).

\subsection{Contracted forms and concord}

In this approach to the nature of the verbal constituents of TCs, questions such as the (non-) use of contracted forms or the presence of number agreement between the verb and the notional subject also merit some attention.

In the TCs analysed here, the use of the contracted forms of modals and semi-modals is basically restricted to speech. All the contracted negative forms of central modals (except cannot) that were found occurred in spoken TCs, and the same happened with the different contracted idiomatic phrases that were registered (cf. Tables 3 and 4). This fluctuation between speech and writing in the use of the different kinds of modal expressions is consistent with the general features of the two media of expression. In this sense, the higher speed of production and the tendency to economy that characterises interactive situations, and speech in general, favour the use of contracted and reduced forms such as 's gonna to, is gotta, etc. (cf. Biber, 1991: 19). Conversely, such forms are almost systematically excluded from the written medium, in which the tendency is rather to benefit full forms over contracted ones, and explicitness over economy of language. Notice the case of the negative forms of can in Table 3, for instance: only two cases were registered, the shorter form can't was used in speech, whereas the longer form cannot was observed in writing.

In connection with this recurrent use of contracted forms in speech, and with the nature of the verbal components of TCs, it was also observed that the form there's is considerably more common in the spoken component of the corpus analysed here. While $44.22 \%$ of the spoken TCs contained this form, only $3.92 \%$ of the written ones incorporated there's.

Moreover, in the analysis of the corpus, a close relationship was found between the use of this contracted form there's and non-concord. That is, on many occasions, the singular verbal form in there's occurred with plural notional subjects -the label non-concord arises then from the traditional consideration that the verb in TCs should agree in number with the real or notional subject-. Space does not permit a thorough investigation of this relationship here; some further details about concord in PDE TCs can be found elsewhere (cf. Martínez Insua and Palacios Martínez, forthcoming). The relevant finding here is that while nonconcord is present in $9.40 \%$ of $b e$ TCs $-13.29 \%$ of $b e$ TCs in the spoken sample, and $3.40 \%$ of $b e$ TCs in the written one-, it keeps away from the group of non-be TCs, in which none of the examples registered presented overt lack of number agreement between the verb and the notional subject.

There's six of the best (CKG $24 \mathrm{wt}$, leisure).

There's two another two (FMG $1451 \mathrm{sp}$, educational and informative). 
Right, so let's start the structural, now there's a whole range of different types of structural isomers (F7U 241 sp, educational and informative).

It is accepted in this study that this tendency to use the contracted form there's with both singular and plural notional subjects ${ }^{11}$ (like in (26)-(28) above) supports its consideration as an introductory presentational formula, or "presentative signal" (Breivik, 1990: 153). This characterisation of there as a presentative signal will be further examined in section 6 below, where it will be maintained that it is precisely this inherent signalling function of there that is shared by both be and non-be TCs.

Finally, and referring once more to the use of contracted forms, it was also in the spoken sample that the only six cases of the contracted plural form there're were found. There're is a considerably marginal form, probably due to the fact that its possible phonetic realisations - $/ \mathrm{DW}^{\mathrm{r}} /$ or $/ \mathrm{D}^{\mathrm{W}} \mathrm{r} /$ - may be easily confused with the weak form of they' $r e / \mathrm{D}^{\mathrm{W}} \mathrm{r}$ / (cf. Wells, 1990).

er but there're some really nifty ones in the Chemistry Department which cou Doug could borrow for an hour and a half of an evening. (FM2 2331 sp, business).

But really these people, and there're about thirty other nationalities, by the way, being held, he just wants to show how though he is, and it's useful in the future, as now, when he wants to make a gesture. (HM4 $613 \mathrm{sp}$, leisure).

There're no additives? (G43 126, sp, public and institutional).

There're no additives in them at all. (G43 127, sp, public and institutional).

On the basis of the findings presented here and without forgetting their limitations, we may point out that, in PDE the use of there is not restricted to signal (non-)existence when it cooccurs with the verb be. Rather, its use seems to be expanded so as to convey its signal function also when co-occurring with other-than-be verbs. The slightly higher frequency of non- $b e$ TCs found in the spoken sample lend further credence to the statement that this use of there, with other functions rather than the expression of (non-)existence, is more common in interactive spoken texts than in written ones. Also, as claimed by Biber et al. (1999: 487), "it is not surprising to find linguistic novelty establishing itself in conversation first, and then spreading to the written registers".

No strong claims can be made on this point, however, without paying attention to bigger corpora and to diachrony, especially knowing that, in some previous studies, the distribution of non- $b e$ TCs was attested to be considerably different. See, for instance, Biber et al. (1999) or Breivik (1990), who found that non-be TCs were mainly restricted to the written samples of the corpora they analysed.

Having observed the frequency and distribution of the different verbal forms in the corpus under analysis, it is appropriate now to concentrate on the similarities and the differences between $b e$ and non-be TCs, regarding formal and functional aspects. 


\section{The formal features of non-be TCs and $b e$ TCs}

Focusing now on the structural features of the TCs analysed, I will try to observe to what extent -if any- be TCs and non-be TCs differ from each other, as far as their structure is concerned.

As seen in Table 1 above, the number of non-be TCs in the corpus of PDE analysed here amounts to 10 . The first thing that deserves our attention is the type or types of verbs they contain. As generally pointed out in the literature, the majority of the other-than-be verbs found in the analysis are intransitive verbs of movement (follow, come) or be-like verbs and verbs of permanence (seem/remain). However, there is also an example that contains what might be considered as a pseudo-intransitive verb (cf. Crystal, 1997: 397) in example (5), repeated here as (33) for convenience.

Oh yes varied, and there interesting, with, with children, particularly if they erm if I'm doing workshops er with art and writing workshops and we come across an abstract picture, the children music, and, we, as a way into their vocabulary which isn't as large as their, their writing skills, and there'll, there'll decipher an abstract picture, wonderful language and I like doing that you know (F71 $110 \mathrm{sp}$, educational and informative).

The use of decipher as the main verb of a TC is certainly unexpected given the transitive nature of this verb and the tendency for there to combine only with intransitive verbs. In addition to that, when presented to native informants, example (5) has been considered as considerably marginal, if not completely unacceptable, and it is true that it rather seems to be a case of misspelling (for they'll decipher). Nevertheless, from a corpus-driven perspective, this example should not, in principle, be disregarded. The presence of decipher might find an explanation in the mentioned pseudo-intransitive uses of verbs. The case we are facing here might be one of those marginal uses of certain verbs that, in spite of being prototypically transitive, function as intransitive in certain contexts. Thus, decipher is a verb that usually takes two dependent elements of structure (subject and object) but in this particular case, the agent is assumed (the children), and the only dependent element remains the post-verbal NP that would function as direct object under normal circumstances (an abstract picture). Like in other cases of pseudo-intransitive verbs, and unlike prototypical intransitive constructions, an agent transform (Crystal, 1997: 397) is possible (the children will decipher an abstract picture), and the result is marginal both to transitive and intransitive constructions.

With regard to the syntactic structures of the non-be examples, we observe that in general they follow the main tendencies observed in be TCs. Some of them are minimal TCs, meaning that their notional subjects lack any kind of complementation or postmodification, like examples (3), (4) -and also (5) if we should consider it as a valid example. Some other TCs contain postmodifiers within their PVNPs themselves, such as prepositional complements or relative clauses, like examples (2), (7) and (9). Finally, there are also examples of TCs with some kind of adverbial postmodification outside the PVNP itself, often co-occurring with other complements in the PVNP, like in examples (1), (6) 
(8), and (10). ${ }^{12}$ It is relevant that, despite the fact that the number of examples we are dealing with is very small, the observation of the subjects in the TCs under analysis seems to evince the existence of certain collocations. This is observed, for instance, in the tendency that there + come shows to collocate with the nouns point and time.

The subjects of the non-be TCs analysed do not differ in any significant way from the subjects normally found in $b e$ TCs. All of them, except one, are prototypical indefinite NPs, and the only case of notional subject containing the definite article the (in (7) above) is perfectly acceptable if adequately contextualised. As advanced by Abbott (1993:52), the acceptability or non-acceptability of such TCs should not be approached from a formal perspective, since "the role of context is crucial in predicting what kinds of NPs can occur in there-constructions as well as the restrictions that exist".

In the case of the notional subjects containing point and time, given the rather general meaning of these nouns, a postmodification is required, and actually given in most cases. In general, relative clauses and prepositional complements are frequently chosen as postmodifying structures in this kind of TC. Finally, as mentioned above, it is noteworthy that no cases of non-concord were observed in the examples of non-be TCs under analysis. In some of these examples -(1), (2), (5), (6)-, the presence of a modal auxiliary before the main verb blocks the existence of an overt mark of concord (cf. Biber et al., 1999: 180; Quirk et al., 1985: 756), but concord is maintained in the rest of the cases. The presence of modals in non-be TCs will be discussed in the approach to the communicative function of such statements, in section 6 below. Also in the following paragraphs, I will look at the possibility that these non-be TCs were rephrased as be TCs, and the communicative consequences that such change would convey.

\section{The pragmatics of $b e$ and non-be TCs}

The pragmatics of TCs has been discussed at length in literature in the last few decades, which mainly focused on the explanation of TCs with definite PVNPs. As a part of a more extensive study of TCs in PDE, the approach adopted here differs from previous accounts in the sense that it does not focus exclusively on so-called definite TCs. It attempts to classify TCs from a pragmatic perspective, going beyond the general acceptance that their communicative role is to draw the addressee's attention towards the (non-)existence of some entity. On such a basis, it is hypothesised that TCs can be used with a number of communicative purposes besides that of signalling or introducing a given entity and asserting its existence. In brief, the main assumption is that TCs are liable to convey both retrospective and prospective functions in discourse. TCs with retrospective functions maintain a close relationship with the previous context or refer back to it. Those with prospective functions expand the message forward, providing completely new information or further developing already given information (for further details on this classification of the pragmatic functions of PDE TCs, see Martínez Insua, in press). 
Concentrating now on non-be TCs, as previously advanced, it is proposed here that even though they cannot be claimed to express existence in a strict sense, they do perform parallel communicative functions to those performed by be TCs. It is acknowledged that, as suggested in previous research (Breivik, 1990: 159, 175), there functions as a presentative signal, and attracts the addressee's attention to a given entity, wherever it occurs, either in be or in non-be TCs. Granted that, however, it is also maintained that a main difference between be and non-be TCs can be found in the different scopes that the signal function of there has in one and the other.

Thus, in be TCs the use of a "transparent" verb such as be helps the speaker/writer to draw the addressee's attention to the logical subject, signalling its newness, and simply presenting it as existing. Similarly, in non- $b e$ TCs, the addressee's attention is once more drawn towards the notional subject. However, in this case, it is not merely presented as existing, but rather as entering into the scene of discourse (come), remaining in it (remain), coming after some previous event -follow-, etc. Under such circumstances, the existence of the notional subject in non-be TCs seems to be necessarily presupposed or unquestionable for the speaker/writer, who assumes that this will also be the case for the addressee. The speaker/writer's intention is then to go one step further, and highlight not only the existence of some entity, but also its being involved in some given process -once its existence is taken for granted-.

From another perspective, Birner and Ward $(1996,1998)$ claimed that having be or another verb implies that the PVNP in TCs will be sensitive to different discourse constraints. The PVNP of existential TCs (be TCs) must present hearer-new information, while the PVNP presentational TCs (non-be TCs) are not restricted to the presentation of hearer-new information. The entities they present may be also hearer-old, provided they are discourse-new. ${ }^{13}$ Almost every non-be TC in the present study contains an indefinite PVNP. Applying Birner and Ward's arguments, this would mean that the entities they represent are both, discourse-new and hearer-new, at the same time. In the case of the definite PVNP (7), although the entity presented (the possibility) is treated as hearer-old, it is discourse-new at the point in which it is introduced into the text.

It is relevant that, in the non-be TCs found in the spoken sample, the use of verbs otherthan-be is significantly linked to the use of modals, semi-auxiliaries and fixed idiomatic phrases. As seen in the tables presented above, the presence of modal expressions in TCs does not usually exceed $10 \%$. Therefore, the marked frequency of such expressions in nonbe TCs is probably evincing certain features of the communicative value of such TCs. Carrying modality, these TCs convey a more evident expression of the speaker's attitude towards the message (consider, for instance, the effects of must in (6) above). In some cases, this modality is further reinforced with the use of adverbials such as surely (3), or introductory clauses such as I think that (4). It might be said then that, in the corpus under analysis, the speaker/writer's attitudes tend to be more perceptible in non-be TCs, specially in the spoken sample. ${ }^{14}$

To finish this section I will refer to the fact that most of the non-be TCs analysed here could be rephrased as be TCs from a formal point of view. However, such substitution 
would imply the loss of certain semantic nuances and communicative values. The achievement of such nuances and values (which was presented above as the main differentiating factor between be and non-be TCs) is here presumed to be the reason that leads the speaker/writer to choose the there-pattern and use it with a verb other than $b e$. Thus, if (1)-(10) were rephrased as be TCs, part of the semantic and communicative nuances conveyed by the non-be TCs would be lost as a consequence. The speaker/writer's intention to signal not only the notional subject but also the verbal constituent would be lost or bleached.

To illustrate this briefly, TCs with come as their main verb (examples (19)-(4) above) not only take for granted that a certain time or point exists. They also seem to imply that such moment/point is not exactly present in the scene of discourse at that very moment, but will eventually arrive or come to it, once certain circumstances are fulfilled. The TC with follow (example (8) above) not only presents the existence of a given set of years (there followed lean years...), but rather states that such years came after something that has been necessarily mentioned or referred to in the pre-co(n)text. It presents such years as a consequence or result of some other event or circumstance, thus strengthening the linkage between this TC and its previous context.

A similar reasoning can be offered for the TCs with remain and develop. Remain (in example (7) above) contains the semantic nuance of "permanence, lasting in time", and it is this meaning that the TC as a whole gains from its use. The writer does not present the possibility as a completely new entity that exists. $\mathrm{He} / \mathrm{sh}$ presents it rather as the one that is left from a set that must be necessarily known by the addressee (a communicative implication that is implemented with the use of the definite article the). Likewise, develop (example (9) above) is not merely expressing the existence of the debate. Using this verb, the writer leaves the addressee with the impression that the debate appeared gradually, that it did not emerge all of a sudden. In example (10), the use of seem provides the statement with a greater amount of subjectivity than the use of $b e$ would imply. In all the cases, the use of a construction with there allows speakers and writers to signal and highlight not only the existence of the notional subject, but also the process through which such logical subjects come into existence or in which they are involved.

As mentioned, the case of decipher is considerably peculiar and stylistically marked. It is one of the very few cases of TCs with a transitive verb used as a pseudo-intransitive verb. Even though this TC and its meaning are not prototypical, the signal function of there might be still observable. Like in the rest of the cases, not only the notional subject (an abstract picture), but also the main verb-decipher-, would be highlighted, and the addressee's attention drawn towards both of them. Conversely, the agency of the process would be silenced conferring a particular depersonalised quality to the statement. In this particular case, there do not seem to be any active paraphrases of the sequence as a $b e \mathrm{TC}$, which does contributes to its oddity. 


\section{Conclusions}

The claim that $b e$ is the verb par excellence of English TCs has been amply supported with the data obtained in the present study. Such data have also attested that other lexical verbs may occur in TCs. All the non-be TCs under analysis contain verbs that express notions of movement (come), entrance into the scene of discourse (come, develop, decipher), being and permanence (remain) or continuation (follow). As hinted above, even decipher, might be considered to be used as a pseudo-intransitive verb in (5), possibly carrying that notion of emergence, in the sense that deciphering an abstract picture can be said to involve the disclosure of a more discernible explanation or understanding of the picture. It would be this appearance of a clearer understanding of the picture what makes the TC feasible for use.

It is generally admitted that $b e$ and other vacuous intransitive verbs are preferred in TCs because of the necessity that these constructions have to dispense with any element that could eclipse the intended denotation present in them. This conclusion implies the consideration of there as an item that simply marks existence and that, from a semantic point of view, the post-verbal constituent is the only significant segment in the construction. Nevertheless, the data collected have shown that the presence of verbs other than be in TCs does not imply an eclipse of their intended denotation, but rather an extension of their signal and highlighting function to affect both the notional subject and the verb.

The possible effects of the variant of medium of expression (written vs. spoken) on the choice of the verb for the TC turned out to be rather limited in the corpus analysed here. Be is predominant in the written sample and also in the spoken one, while a slightly higher frequency of non-be TCs was registered in spoken TCs $(0.42 \%$ in the spoken sample vs. $0.27 \%$ in the written one). Likewise, modals, catenative expressions, marginal auxiliaries and fixed verbal expressions are slightly more frequent in written TCs, although the difference is not really important.

A number of circumstances were referred to with the ultimate aim to characterise there as a formula with a signal force that serves speakers and writers not only to present more or less new entities as existing, but rather to attract the addressee's attention towards them -in $b e$ TCs-, and possibly also to the process in which they are involved -in non-be TCs-. The fact that the spoken sample presented higher frequencies of TCs, of non-be TCs, of the contracted formulaic forms there's and there're, or even of singular agreement with plural notional subjects, was taken as evidence that the TC is actually a relatively productive pattern used by speakers for certain pragmatic and communicative purposes, even if the notion they try to emphasise is not strictly speaking one of existence.

\section{Questions for further research}

At this point, we can say that English TCs constitute a complex linguistic phenomenon in which semantic, pragmatic and syntactic aspects show a complicated interaction. In the light of such argument, the present study has sought to approach the nature of their verbal 
component considering not only structural, but also semantic and pragmatic facts. At different points of the analysis, and in connection with this interaction, I referred to certain questions that were, however, necessarily left open to further research.

Chiefly, it will be interesting to test the existence of an association between non-be TCs, modals and semi-modals, and medium of expression. Besides that, the higher frequency of non-be TCs in speech that has been attested in the present study should be confirmed with the analysis of bigger corpora, if we want to develop some reliable hypothesis about a potential ongoing expansion of the there-pattern for pragmatic reasons.

\section{Notes}

1. This research has been funded by the Galician Ministry of Education (Secretaría Xeral de Investigación PGIDT00PXI20407PR). This grant is hereby gratefully acknowledged. The present paper is a revised and expanded version of an initial exploratory study of the verbs in Present Day English (PDE) there-constructions (TCs), which was presented at the 23th AEDEAN (Spanish Association of Anglo-American Studies) international conference (held in December 1999).

2. From this perspective, it is claimed that in those analogical constructions to the existential pattern that contain full lexical verbs "no pragmatic effect is intended" (Pérez Guerra, 1999: 86).

3. Being corpus/data-driven, (Stubbs, 1993), this study will seek to draw its conclusions from the unbiased study of the data, rather than seeking in the data a confirmation of pre-assumed claims and conclusions.

4. The alphanumerical code which appears after most of the examples cited in the paper refers to the text and the lines of the British National Corpus (hereafter BNC) from which the example has been taken. Wt. stands for written, and $s p$. stands for spoken. The rest of the information provided refers to the genre or text type to which the example belongs. Thus, example (1) was drawn from the spoken text identified with the code K6A. The TC under analysis (with existential there and the verbal unit marked up in bold characters) is inserted in the linguistic sequence numbered as 435 . Finally, the last label provided between brackets (leisure) means that text K6A shares a number of its main features with a group of other texts that the compilers of the BNC decided to label as leisure, on the grounds of their subject matter and contents.

5. Internationally recognised as a reliable source of linguistic data (Biber et al., 1999: 27), the BNC contains a total of about 100 million words of Standard English (over 10 million words of spoken English and around 90 million words of written English), more than 6 million sentences, taken from more than 4000 different texts carefully selected.

6. Both labels, register and genre, will be used here to refer to any distinguishable variety of language that has achieved a level of general recognition, either in speech or writing, and that may be defined according to its use in social situations. That is, a variety imposing identifiable characteristics on the use of language, in relation to subject-matter, purpose, textual structure, form of argumentation and level of formality (Crystal, 1997: 168; Biber et al., 1999: 15).

7. The BNC is generally characterised as a sample corpus (its samples do not generally exceed the 45000 words), a synchronic corpus (containing samples of English from the year 1960), a general corpus (not restricted to any particular subject field, register or genre), a monolingual corpus (in the sense of containing only British English), and a mixed corpus (with both spoken and written English). 
8. The genres identified in the written sample are: Imaginative, Arts, Belief and Thought, Commerce, Leisure, Natural Science, Applied Science, Social Science and World Affairs. In the spoken sample, the labels provided in the BNC are: Educational and informative, Business, Institutional, and Leisure

9. The terms Semi-auxiliaries and modal idioms are the labels used by Quirk et al (1985, but other terms referring to these same expressions are semi-modals, quasi-modals and periphrastic modals (cf. Biber et al (1999: 484).

10. Notice that, in the corpus analysed here, catenative constructions were only attested in be TCs.

11. Singular agreement was found to be very common in the TCs analysed here, specially in the spoken sample. $66.28 \%$ of the total present singular agreement: $71.40 \%$ of the spoken TCs, and $58.54 \%$ of the written ones.

12. The modifiers in the post-verbal sequences of TCs were classified as somewhat peripheral -adverbials and locatives-, or integrated in the structure of the PVNP -prepositional complements, relative clauses, etc.-- previous classifications given by Grzegorek (1984: 147) and Biber et al. (1999: 949) were taken as guidelines.

13. According to the classification that Ward and Birner $(1994 ; 1995)$ provide, the category of discourse-new entities includes a number of definite PVNPs designating "hearer-old entities marked as hearer-new", "hearer-old entities newly instantiating a variable" or even "false definites"(cf. Ward and Birner, 1994: 171-ff for a definition of these labels.

14. This connection between the use of non- $b e$ TCs, the expression of modality and the medium of expression is left here as a question for further research. Due to space constraints, going into further details about this point is not possible in this paper.

\section{References}

Abbott, Barbara (1993): Definiteness, existentials, and the "List" interpretation in Working Papers in Linguistics, 40 (SALT II Proceedings from the Second Conference on Semantics and Linguistic Theory): 1-16.

Biber, Douglas (1991): Variation across Speech and Writing. Cambridge: Cambridge University Press.

Biber, Douglas, Stig Johansson, Geoffrey Leech, Susan Conrad and Edward Finegan (1999): Longman Grammar of Spoken and Written English. London: Longman.

Birner, Betty J. and Gregory Ward (1996): "A Crosslinguistic Study of Postposing in Discourse". Language and Speech. 39 (2-3): 113-142.

. (1998): Information Status and Noncanonical Word Order in English. Studies in Language Companion Series, 40. Amsterdam/Philadelphia: John Benjamins Publishing Company.

Bolinger, Dwight (1977): Meaning and Form. London: Longman, English Language Series 11. Breivik, Leiv Egil.1990): Existential There. A Synchronic and Diachronic Study. Studia Anglistica Norvegica, 2. Oslo: Novus Press ( $2^{\text {nd }}$ edition).

Butler, Christopher (1985): Statistics in linguistics. Oxford: Basil Blackwell Ltd.

Crystal, David (1997): A Dictionary of Linguistics and Phonetics. Oxford: Blackwell Publishers Ltd. (4 $4^{\text {th }}$ edition).

Curme, George O (1966): English Grammar. New York: Barnes and Noble, Inc. 
Davidse, Kristin (1992): "Existential constructions: a systemic perspective". Leuvense Bijdragen, 81: 71-99.

Downing, Angela. and Phillip Locke (1992): A University Course in English Grammar. London: Phoenix Elt.

Erdmann, Peter (1976): There Sentences in English. München: Tuduv Studie.

Grzegorek, Maria (1984): "English sentences with introductory there and their Polish counterparts". In J. Fisiak, ed. Contrastive Linguistics: Prospects and Problems. Berlin: Mouton Publishers. 143-169.

Hannay, Michael (1985): English Existentials in Functional Grammar. DordrechtHolland/Cinnaminson-U.S. A.: Foris Publications.

Huddleston, Rodney (1984): Introduction to the Grammar of English. Cambridge: Cambridge University Press.

Martínez Insua, Ana E. (in press): "Present Day English existential there-constructions and their pragmatics. Towards an integrated categorisation" (to appear in Miscelánea).

Martínez Insua, Ana E. and Ignacio M. Palacios Martínez (forthcoming): "A corpus-based approach to non-concord in present day English existential there-constructions" (to appear in English Studies).

McNally, Louise (1997): A Semantics for the English Existential Construction. New York and London: Garland Publishing, Inc.

Milsark, Gary. L (1979): Existential Sentences in English. MIT Dissertation. (Mimeographed by the Indiana University Linguistics Club). Published in the series Outstanding Dissertations in Linguistics.

Pérez Guerra, Javier (1995): "THERE in Late Middle English and Early Modern English". In B. Santano Moreno, A.R. Birtwistle and L.G. Giron Echevarrís, eds. Papers from the VII International Conference of the Spanish Society for Medieval English Language and Literature. Universidad de Extremadura. 217-237.

(1999): Historical English Syntax. A Statistical Corpus-Based Study on the Organisation of Early-Modern English Sentences. Muenchen: Lincom Europa.

Quirk, Randolph, Sidney Greenbaum, Geoffrey Leech and Jan Svartvik (1985): A Comprehensive Grammar of the English Language. London and New York: Longman.

Sinclair, John M (1992): "Trust the text: the implications are daunting". In M. Davies, and L. Ravelli, eds. Advances in Systemic Linguistics. Recent Theory and Practice. London and New York: Pinter Publishers. 5-19.

Stubbs, Michael (1993): "British Traditions In Text Analysis - From Firth To Sinclair". In M. Baker, G. Francis, and E. Tognini-Bonelli, eds. Text and Technology. In Honour of John Synclair. Philadelphia/Amsterdam: John Benjamins. 1-36.

Ward, Gregory and Betty J. Birner (1994): "English there-sentences and information status". Proceedings of the Israeli Association of Theoretical Linguistics Ninth Annual Conference and Workshop on Discourse.

Wells, John Ch (1990): Longman Pronunciation Dictionary. Hong Kong: Longman. 